\title{
Caloplaca sol (Teloschistaceae), a new coastal lichen from Great Britain
}

\begin{abstract}
Alan ORANGE
Abstract: Caloplaca sol is described as a new species from limestone and basic siliceous rocks on the southern and western coasts of Great Britain. It is characterized by a well-developed, crustose, nonplacodioid, epilithic, cracked, orange-yellow thallus, almost concolorous apothecia up to $0.66 \mathrm{~mm}$ diameter, and ascospores c. $11 \cdot 0-12 \cdot 2-13.0 \mu \mathrm{m}$ long with a septum $c$. $0.4 \times$ the ascospore length. Caloplaca dalmatica is related but differs in the endolithic or only thinly epilithic thallus. Caloplaca marina is darker orange in colour, with more convex areoles, and is mostly confined to the splash zone of the seashore. Caloplaca maritima differs in the typically more convex, sometimes isolated areoles, and often in the presence of a crenulate thalline margin in young apothecia. Caloplaca itiana is newly reported from Great Britain from coastal limestone; it differs from $C$. sol in the thallus being endolithic or almost so, and from $C$. dalmatica in the more completely endolithic thallus and the larger ascospores.
\end{abstract}

Key words: Flavoplaca, France, key, maritime, taxonomy, Wales

Accepted for publication 16 November 2017

\section{Introduction}

Species of Caloplaca in the broad sense often form a conspicuous part of the lichen communities in which they occur due to the yellow to orange colour of the thallus or apothecia in many taxa. The species-level taxonomy in Europe has changed significantly in recent years due to more detailed studies, supported by DNA sequencing, which have revealed additional taxa within formerly more broadly defined species or species-groups, including C. cerina (Šoun et al. 2011), C. citrina (Arup 2006; Vondrák et al. 2009), C. crenulatella (Vondrák et al. 2011), C. herbidella (Arup \& Åkelius 2009) and C. holocarpa (Arup 2009; Vondrák et al. 2012, 2016). Traditionally, growth habit was used as a major generic character in Teloschistaceae and the genus Caloplaca was restricted to species with crustose or placodioid thalli. Caloplaca in this sense is now known to be non-monophyletic (e.g. Gaya et al. 2012). Recently, a new arrangement of the family has been proposed in which

A. Orange: Natural Sciences, National Museum of Wales, Cardiff CF10 3NP, U.K. Email: alan.orange@ museumwales.ac.uk
Caloplaca is restricted to 12 species related to the type of the genus, $C$. cerina, and the remaining species are distributed throughout numerous resurrected or newly described genera (Arup et al. 2013).

A conspicuous unidentified species of Caloplaca in the broad sense has been known on coastal rocks in Great Britain for many years and is described below. It is related to, among others, the species currently known as C. dalmatica in Great Britain. The relationship of these species to the recently described C. itiana was investigated, with the result that C. itiana is newly recorded from Great Britain.

\section{Materials and Methods}

Thallus and apothecial sections were mounted in water and $5 \% \mathrm{KOH}(\mathrm{K})$. Ascospores were measured in water. The spores of recently collected specimens were first killed by warming apothecial sections in a drop of water, then allowing them to dry, and then remounting in water. Ascospore measurements for each species are given in the following format (after Frolov et al. 2016): (minimum -$) \bar{x}_{1}-\bar{x}_{2}-\bar{x}_{3}(-$ maximum $)$, where minimum and maximum are extreme values, $\bar{x}_{1}$ is the smallest mean value measured for each individual collection, $\bar{x}_{2}$ is the mean of all values measured in that species, and $\bar{x}_{3}$ is the largest mean value measured for each individual collection. The measurements are followed by the standard deviation (SD) of $\bar{x}_{2}, \mathrm{~N}=$ the total number of 
measurements for all samples of that species and $n=$ the number of samples from which measurements were made.

Recently collected or frozen material was used to generate DNA sequences. BLAST searches (http://blast. ncbi.nlm.nih.gov/Blast.cgi) were used to find sequences similar to those of the new species. Additional possible related taxa, and potential outgroup taxa, were suggested by the analyses in Arup et al. (2013). A sequence of Caloplaca oasis was used as outgroup in the ITS analysis. Specimens used in the analyses are shown in Table 1.

DNA was extracted using the Qiagen DNeasy Plant Mini Kit; the manufacturer's instructions were followed except that warm water was used for the final elution. PCR amplification was carried out using Bioneer AccuPower PCR Premix in $50 \mu \mathrm{l}$ tubes. The two internal transcribed spacer regions and the $5.8 \mathrm{~S}$ region (ITS15.8S-ITS2) of the nuclear ribosomal genes were amplified, using the primers ITS1F and ITS4. The PCR thermal cycling parameters were: initial denaturation for $5 \mathrm{~min}$ at $94^{\circ} \mathrm{C}$, followed by 5 cycles of $30 \mathrm{~s}$ at $94^{\circ} \mathrm{C}, 30 \mathrm{~s}$ at $55^{\circ} \mathrm{C}$ and $1 \mathrm{~min}$ at $72^{\circ} \mathrm{C}$, then 30 cycles of $30 \mathrm{~s}$ at $94{ }^{\circ} \mathrm{C}, 30 \mathrm{~s}$ at $52{ }^{\circ} \mathrm{C}$ and $1 \mathrm{~min}$ at $72^{\circ} \mathrm{C}$. PCR products were visualized on agarose gels stained with ethidium bromide and purified using the Sigma GenElute PCR Clean-Up Kit. DNA sequencing was performed by DNA Sequencing \& Services (MRC I PPU, School of Life Sciences, University of Dundee, Scotland, www.dnaseq. co.uk) using Applied Biosystems BigDye v3.1 chemistry on an Applied Biosystems 3730 automated capillary DNA sequencer. Sequences were assembled and edited using GeneStudio software (http://www.genestudio. com). Alignment was carried out using BioEdit (http:// www.mbio.ncsu.edu/BioEdit/bioedit.html); ClustalW was used to create an initial alignment which was edited manually. Ambiguously aligned regions were deleted before further analysis of the alignment.

ITS sequences were newly prepared for 40 specimens. The ITS alignment was $574 \mathrm{bp}$ in length. A total of $66 \mathrm{bp}$ were excluded from the analysis as they could not be unambiguously aligned (ITS 1: 2 regions with a total of $41 \mathrm{bp}$, ITS2: 3 regions with a total of $25 \mathrm{bp}$ ). After deletion of ambiguous regions, the alignment was 509 characters in length.

Maximum likelihood (ML) analyses were used to investigate phylogenetic relationships and support values. Analysis was performed using RAxML v8.2.10 (Stamatakis et al. 2008; Stamatakis 2014), as hosted on the CIPRES Science Gateway (Miller et al. 2010). PartitionFinder v2.1.1 (Guindon et al. 2010; Lanfear et al. 2012, 2017) was used to optimize partitions and substitution models. Three regions of the ITS (ITS1, 5.8S, ITS2) were used as input. Parameters included linked branch lengths and greedy search, and the '-raxml' command was used to limit the number of models to those used in RAxML. PartitionFinder returned GTR + $\mathrm{G}$ for ITS1 and ITS2, and GTR + G+I for the 5.8S region. Analyses with RAxML used rapid bootstrapping with 1000 iterations and the GTRGAMMAI substitution model; a search for the best-scoring ML tree was carried out with the bootstrap analysis in a single run. The resulting tree was visualized using MEGA v4 (Tamura et al. 2007). Support values of $\geq 70 \%$ maximum likelihood bootstrapping were regarded as significant.

Sequences used in the analysis are shown in Table 1.

Additional figured specimens. Caloplaca marina: Great Britain: Wales: V.C. 41, Glamorgan, Gower, Port Eynon, grid ref. 21/4649.8463, $51.539638^{\circ} \mathrm{N}$, $-04.214855^{\circ} \mathrm{W}$, on limestone in splash zone of seashore, 2014, Orange 21907 (NMW-C.2014.008.60). England: V.C. 9, Dorset, Isle of Portland, below Grove Cliff, grid ref. $30 / 7047.7166,50.543955^{\circ} \mathrm{N},-02.418112^{\circ} \mathrm{W}$, on limestone on rocks on slope above seashore, 2012, Orange 21071 (NMW-C.2012.002.70).

Caloplaca maritima: Great Britain: Wales: V.C. 52, Anglesey, Amlwch, Parys Mountain, grid ref. 23/ $4433.9052,53.388457^{\circ} \mathrm{N}, 04.342421^{\circ} \mathrm{W}$, on rock in mortared wall of disused windmill, 2011, Orange 20529 (NMW-C.2011.014.34); Benllech, Trwyn Dwlban, grid ref. $23 / 5317.8203,53.314746^{\circ} \mathrm{N}, 04.206073^{\circ} \mathrm{W}$, on limestone rocks above seashore, 2012, Orange 21047 (NMW-C.2012.002.66).

\section{Results}

\section{ITS analysis}

The tree resulting from analysis of the ITS1-5.8S-ITS2 region is shown in Fig. 1. All species included in the tree, with the exception of the outgroup, belong to the genus Flavoplaca, as defined by Arup et al. (2013). The lower nodes of the tree are poorly supported. Specimens from Great Britain described below as the new species Caloplaca sol form a well-supported clade of seven sequences showing little variation. A well-supported clade with Caloplaca dalmatica and a single sequence named as C. navasiana is a sister clade to the $C$. sol clade but with very low support. A number of species are not resolved in the tree, being recovered as non-monophyletic. This is likely to be due to a combination of factors including insufficient resolving power of the ITS region between certain closely related species, misidentification of specimens, and insufficient taxon and specimen sampling. For example, five sequences of $C$. maritima form a well-supported clade, but it is nested within a poorly supported clade including $C$. communis and C. havaasii. Another well-supported clade includes sequences named as $C$. calcitrapa, $C$. itiana and C. navasiana, but which are not resolved into individual taxa. It is possible that these three 
TABLE 1. Specimens used in the phylogenetic analysis of Caloplaca species. New sequences are in bold.

\begin{tabular}{|c|c|c|c|}
\hline Species & Country & Voucher & GenBank Accession no \\
\hline Caloplaca arcis & Turkey & Vondrák (CBFS JV5426) & EU563424 \\
\hline C. arcis & Turkey & Vondrák (CBFS JV60936) & EU563438 \\
\hline C. arcisproxima & Ukraine & Vondrák (CBFS 7127) & JF319987 \\
\hline C. arcisproxima & Ukraine & Vondrák (CBFS JV5473) & EU563425 \\
\hline C. austrocitrina & Greece & (PRA JV8712) & $\mathrm{JN} 813423$ \\
\hline C. austrocitrina & Ukraine & Vondrák (CBFS JV5476) & EU563427 \\
\hline C. calcitrapa & Greece & (PRA JV8375) & JN813409 \\
\hline C. calcitrapa & Greece & (PRA JV8729) & JN813413 \\
\hline C. calcitrapa & Spain & Navarro-Rosinés et al. 13387 (Hb. ?) & DQ173227 \\
\hline C. calcitrapa & Bulgaria & Vondrák (CBFS JV5486) & EU563431 \\
\hline C. calcitrapa & Turkey & Vondrák (CBFS JV6100) & EU563444 \\
\hline C. citrina & Sweden & Arup (L03013) & DQ173224 \\
\hline C. citrina & England & Orange 23466 (NMW) & MF595943 \\
\hline C. communis & Turkey & - & KF007913 \\
\hline C. communis & Greece & Vondrák (CBFS JV3763) & EU563410 \\
\hline C. communis & Turkey & Vondrák (CBFS JV6119) & EU563448 \\
\hline C. confusa & Portugal & F. Berger (Hb. F. Berger) & EU563468 \\
\hline C. confusa & France & Hafellner (GZU Haf31862) & EU563457 \\
\hline C. confusa & Bulgaria & Vondrák (CBFS JV3435) & EU563405 \\
\hline C. dalmatica & Wales & Orange 21050 (NMW) & MF595914 \\
\hline C. dalmatica & Wales & Orange 21051 (NMW) & MF595915 \\
\hline C. dalmatica & Wales & Orange 21053 (NMW) & MF595917 \\
\hline C. dalmatica & England & Orange 21057 (NMW) & MF595918 \\
\hline C. dalmatica & England & Orange 21060 (NMW) & MF595919 \\
\hline C. dalmatica & England & Orange 21065 (NMW) & MF595921 \\
\hline C. dalmatica & England & Orange 21067 (NMW) & MF595922 \\
\hline C. dalmatica & England & Orange 21069 (NMW) & MF595924 \\
\hline C. dalmatica & England & Orange 21073 (NMW) & MF595926 \\
\hline C. dalmatica & England & Orange 21076 (NMW) & MF595927 \\
\hline C. dalmatica & Wales & Orange 21766 (NMW) & MF595929 \\
\hline C. dalmatica & Wales & Orange 21803 (NMW) & MF595930 \\
\hline C. dalmatica & Wales & Orange 21808 (NMW) & MF595931 \\
\hline C. dalmatica & France & Orange 23695 (NMW) & MF595945 \\
\hline C. dalmatica & France & Orange 23697 (NMW) & MF595947 \\
\hline C. dalmatica & France & Orange 23699 (NMW) & MF595949 \\
\hline C. dichroa & Sweden & Arup (L 03054) & DQ173229 \\
\hline C. dichroa & Sweden & Arup (L 03108) & DQ173230 \\
\hline C. dichroa & Austria & Hafellner (GZU Haf43519) & EU563458 \\
\hline C. dichroa & Wales & Orange 21811 (NMW) & MF595932 \\
\hline C. dichroa & Wales & Orange 21869 (NMW) & MF595937 \\
\hline C. dichroa & Ukraine & Vondrák (CBFS JV5477) & EU563428 \\
\hline C. flavocitrina & Sweden & Arup (L 04024) & DQ173216 \\
\hline C. flavocitrina & Australia & Kondratyuk 20423 (LD) & KC179365 \\
\hline C. geleverjae & Ukraine & Vondrák (CBFS JV5415) & EU563423 \\
\hline C. havaasii & Norway & Tønsberg 33940 (LD, BG) & DQ647649 \\
\hline C. itiana & Wales & Orange 18786 (NMW) & MF595912 \\
\hline C. itiana & Wales & Orange 21539 (NMW) & MF595928 \\
\hline C. itiana & Wales & Orange 21854 (NMW) & MF595934 \\
\hline C. itiana & France & Orange 23694 (NMW) & MF595944 \\
\hline C. itiana & France & Orange 23696 (NMW) & MF595946 \\
\hline C. itiana & France & Orange 23700 (NMW) & MF595950 \\
\hline C. kantvilasii & Australia & Kondratyuk 20418a (LD) & KC179366 \\
\hline C. limonia & Italy & Poelt (GZU) & EU563467 \\
\hline C. limonia & Turkey & Vondrák (CBFS JV6101) & EU563445 \\
\hline C. marina & Wales & $(\mathrm{BM} 730882)$ & EU563461 \\
\hline C. marina & Wales & Orange 21858 (NMW) & MF595936 \\
\hline C. marina & Wales & Orange 21906 (NMW) & MF595939 \\
\hline
\end{tabular}


TABLE 1 (continued).

\begin{tabular}{|c|c|c|c|}
\hline Species & Country & Voucher & GenBank Accession no \\
\hline Caloplaca marina & Wales & Orange 21910 (NMW) & MF595941 \\
\hline C. maritima & Netherlands & Aptroot 59408 (CBFS JV4987) & EU563451 \\
\hline C. maritima & Wales & Arup L 92256 (CBFS JV4941) & EU563462 \\
\hline C. maritima & Wales & Orange 21047 (NMW) & MF595913 \\
\hline C. maritima & England & Orange 21072 (NMW) & MF595925 \\
\hline C. maritima & Wales & Orange 21903 (NMW) & MF595938 \\
\hline C. microthallina & Wales & Arup L 92307 (CBFS JV4941) & EU563465 \\
\hline C. microthallina & Wales & Orange 17818 (NMW) & MF595911 \\
\hline C. microthallina & France & Orange 23698 (NMW) & MF595948 \\
\hline C. microthallina & $?$ & Sanchez-Biezma et al. (SANT Lich. 9025) & EU639629 \\
\hline C. microthallina & Sweden & Søchting $7480(\mathrm{C})$ & KC179368 \\
\hline C. navasiana & Greece & (PRA JV8871) & JN813402 \\
\hline C. navasiana & Greece & (PRA JV5939) & JN813430 \\
\hline C. navasiana & Italy & Poelt, 1992 (LD) & KC179369 \\
\hline C. nigromarina & Bulgaria & Vondrák (CBFS JV4983) & EU563418 \\
\hline C. nigromarina & Georgia & Vondrák (CBFS JV6091) & EU563436 \\
\hline C. oasis & Russia & Hermansson L-10641 (private hb.) & FJ346544 \\
\hline C. ora & France & Lambinon et al. (W-15494) & HQ234602 \\
\hline C. sol & Wales & Orange 21052 (NMW) & MF595916 \\
\hline C. sol & England & Orange 21062 (NMW) & MF595920 \\
\hline C. sol & England & Orange 21068 (NMW) & MF595923 \\
\hline C. sol & Wales & Orange 21848 (NMW) & MF595933 \\
\hline C. sol & Wales & Orange 21855 (NMW) & MF595935 \\
\hline C. sol & Wales & Orange 21909 (NMW) & MF595940 \\
\hline C. sol & Wales & Orange 21916 (NMW) & MF595942 \\
\hline C. sp. & Spain & Arup L 07151 (LD) & KC179370 \\
\hline C. tavaresiana & Spain & Llimona et al. (BCN) & KC179371 \\
\hline
\end{tabular}

taxa are conspecific but a cautious approach is taken here (see discussion under C. itiana below). A further well-supported clade comprises Caloplaca marina (four sequences as a well-supported subclade) and $C$. nigromarina (two sequences as a well-supported clade), but also with $C$. confusa and $C$. microthallina recovered as non-monophyletic. Resolution between some of these species is likely to require additional gene regions.

\section{The Species}

\section{Caloplaca sol Orange sp. nov.}

\section{MycoBank No.: MB 823252}

Member of the Flavoplaca clade, on limestone and basic
siliceous rocks on the sea coast, with a well-developed,
crustose, non-placodioid, epilithic, cracked, orange-
yellow thallus, almost concolorous apothecia up to
$0.66 \mathrm{~mm}$ diam., and ascospores c. $11 \cdot 5-12 \cdot 0-12.4 \mu \mathrm{m}$
long with a septum $c .0 \cdot 4$ of the ascospore length.
Type: Great Britain, Wales, V.C. 52, Anglesey,
Benllech, grid ref. $23 / 5246.8237,53.317545^{\circ} \mathrm{N}$, $04.216396^{\circ} \mathrm{W}$, on limestone on vertical cliff at top of seashore, 18 April 2012, Alan Orange 21052 (NMW-C.2015.005.37-holotype; MARSSJ-isotype; GenBank Accession MF595916).

(Fig. 2A-F)

Prothallus scarcely visible as a separate structure (fimbriate yellow margins seem already to be lichenized). Thallus epilithic, well developed, up to $280 \mu \mathrm{m}$ thick, orangeyellow; margin well defined, very thin, often fimbriate, young thallus rapidly thickening, forming convex bumps that are rapidly separated by cracks, mature areoles completely or incompletely surrounded by cracks, plane, uneven or gently convex; young thallus orange-yellow, older parts orange-yellow or yellow, or yellow with white mottling (possibly due to damage to cortex). Photobiont cells 6.0-16.5 × 7.5-14.5 $\mu \mathrm{m}$.

Apothecia sparse to numerous, mostly not contiguous, to $0.60-0.66 \mathrm{~mm}$ diam., becoming 
sessile early in development, constricted at base when mature; proper margin yelloworange; disc slightly darker, plane to slightly convex, smooth or slightly yellow-pruinose when young; thalline margin at most weakly developed (Fig. 2F), usually invisible from above, confined to underside of apothecium, paler than proper margin. Hymenium $100 \mu \mathrm{m}$ high. Paraphyses c. $1.2 \mu \mathrm{m}$ near base, with the uppermost cells swollen to $5 \cdot 0-7 \cdot 4 \mu \mathrm{m}$ wide. Asci 8-spored. Ascospores (9.8-)11.512.0-12.4(-14.4) × (5.7-)6.4-6.6-6.9(-7.4) $\mu \mathrm{m}$, SD $\bar{x}_{2} \quad 1.05$ and 0.60 , respectively; length/ width ratio (1.3-)1.7-1.8-1.9(-2.4), SD $\bar{x}_{2}=0.23$; septum (3.7-)4.6-4.9-5.2(-6.6) $\mu \mathrm{m}$, $\mathrm{SD} \bar{x}_{2}=0 \cdot 65$, septum/ascospore length $=(0 \cdot 3-)$ 0.4-0.4-0.4 (-0.5), SD $\bar{x}_{2}=0 \cdot 05 . \mathrm{N}=30, n=3$. Pycnidia not seen.

Chemistry. All yellow parts with unidentified anthraquinones, presumed to be mainly parietin.

Etymology. From the Latin 'sol' (sun), as a noun in apposition, suggested by the rich yellow, often circular thalli and the habitat on dry, sunny rocks.

Ecology and distribution. On vertical or overhanging, dry, sunny faces on limestone or base-rich siliceous cliffs and boulders. Relatively few collections are known but it has probably been overlooked or confused with other species. All the known localities are near to the coast, in south and west Britain from Dorset and Cornwall to North Wales. One specimen (Orange 22749) was collected in small quantity on a cliff $5 \mathrm{~km}$ from the present seashore but the locality is beside Traeth Mawr, a former arm of the sea cut off by a sea wall in 1810, and it is possible that the colony is a relict from that time.

Notes. This species has a well-defined, cracked-areolate, completely epilithic thallus, with more or less concolorous sessile apothecia. Although it is a striking species in the field, it is likely that it has been overlooked as a morph of Caloplaca marina. However, $C$. marina is normally darker orange in colour (Fig. 3A-F; Fig. 3B shows both species growing together) and the areoles are more convex; this species usually grows in the splash zone of the seashore but occasionally in small quantity on rocks well above the high-water mark on slopes facing the sea. Specimens of C. marina from the shore often have a thin, narrow margin to the thallus which rapidly thickens to form thick, strongly convex areoles (Fig. 3F) but those specimens found occasionally above the splash zone may show a more gradual thickening, as in C. sol (Fig. 3A). Individual specimens in herbaria, collected above the splash zone, might be difficult to distinguish by morphology. Caloplaca maritima differs in the more convex areoles, which have a tendency to become isolated as the thallus ages (Fig. 2G \& H), and the young apothecia often have a yellow crenulate thalline margin projecting beyond the proper margin when viewed from above. Caloplaca flavovirescens has large (often to $1 \mathrm{~mm}$ diameter) orange apothecia that contrast strongly in colour with the yellow epilithic thallus. Caloplaca diffusa is often found on damp or poorly drained surfaces and has not been found in coastal habitats in Great Britain; the epilithic thallus is less well developed and the ascospores have a much narrower septum, usually less than 0.33 of the spore length. Caloplaca vitellinula in the sense of Arup (2009) is a non-maritime species, typically with a thin, inconspicuous, discontinuous pale yellow thallus and small apothecia up to $0.44 \mathrm{~mm}$ diameter in British material.

Additional specimens examined. Great Britain: Wales: V.C. 41, Glamorgan, Gower, Southgate, grid ref. 21/ $5587.8689,51.562355^{\circ} \mathrm{N}, 04.080586^{\circ} \mathrm{W}, 2014$, Orange 21848 (NMW-C.2015.005.43); Gower, Southgate, grid ref. $21 / 5587.8685,51.561828^{\circ} \mathrm{N}, 04.080399^{\circ} \mathrm{W}, 2014$, Orange 21855 (NMW-C.2015.005.45); Gower, Port Eynon, Port-Eynon Point, grid ref. 21/4667.8447, $51.53823^{\circ} \mathrm{N}, 04.212285^{\circ} \mathrm{W}, 2014$, Orange 21909 (NMWC.2015.005.46); Gower, Middleton, Mewslade, grid ref. $21 / 4221.8737,51.563076^{\circ} \mathrm{N}, 04.277809^{\circ} \mathrm{W}, 2014$, Orange 21916 (NMW-C.2015.005.47). V.C. 48, Merioneth, Garreg, Garregelldrem, grid ref. 23/6149.4314, $52.967528^{\circ} \mathrm{N}, \quad 04.064065^{\circ} \mathrm{W}, 2015$, Orange 22749 (NMW-C.2015.005.61). V.C. 52, Anglesey, no locality or date, F. E. Griffith (BM). England: V.C. 1, West Cornwall, Kynance Cove, between Gew-graze and Kynance Cove, 1986, P. W. Fames (BM); same locality and date, 1986, P. W. Fames (BM). V.C. 5, North Somerset, Minehead, Greenaleigh Point, grid ref. 21/95.48, 


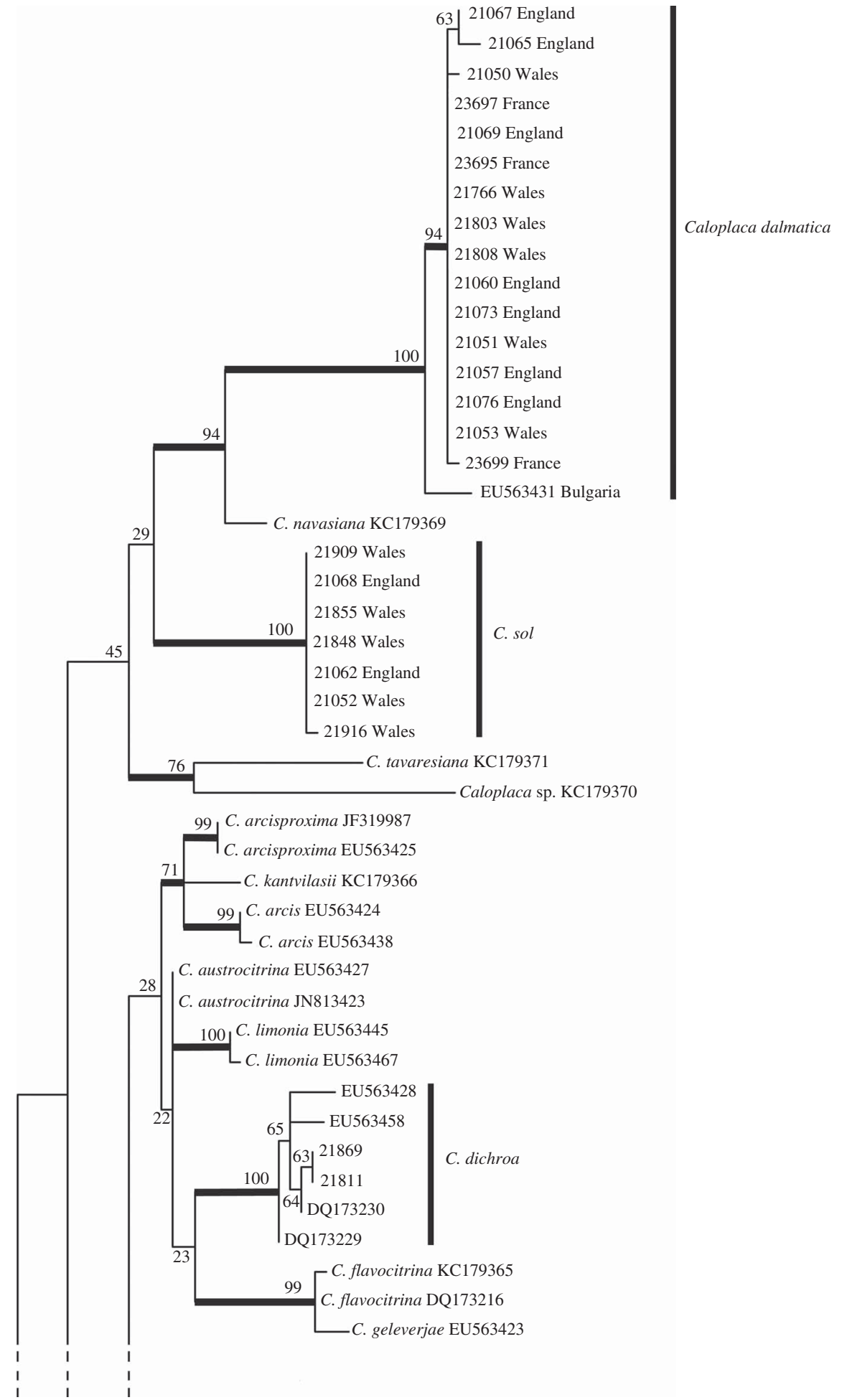

FIG. 1. Continued on facing page. 


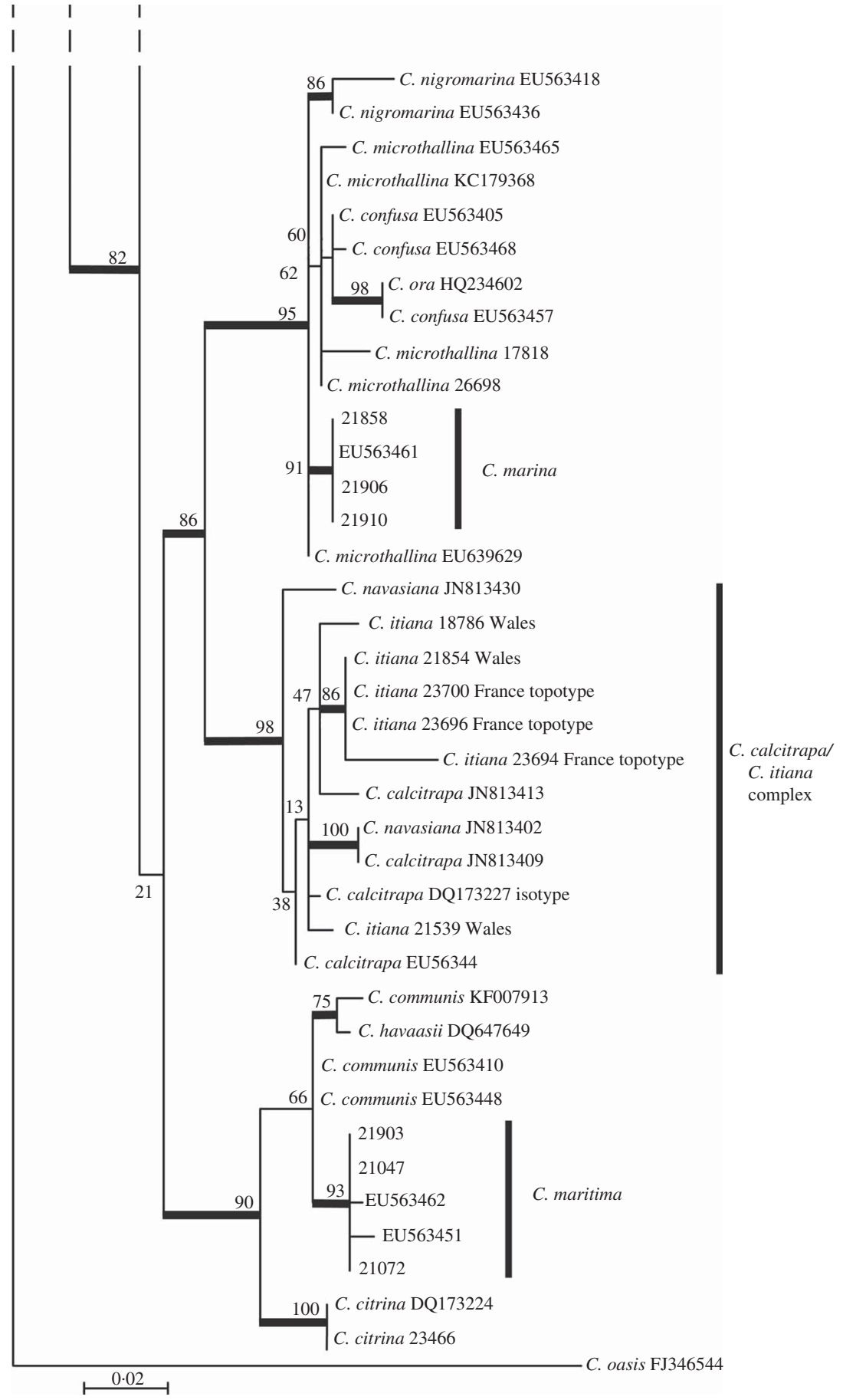

FIG. 1. Phylogenetic relationships amongst Caloplaca species, based on a maximum likelihood analysis of the nuclear ribosomal ITS1-5.8S-ITS2 region. The tree was rooted using Caloplaca oasis. Branches in bold indicate a maximum likelihood bootstrap support of $\geq 70 \%$. 

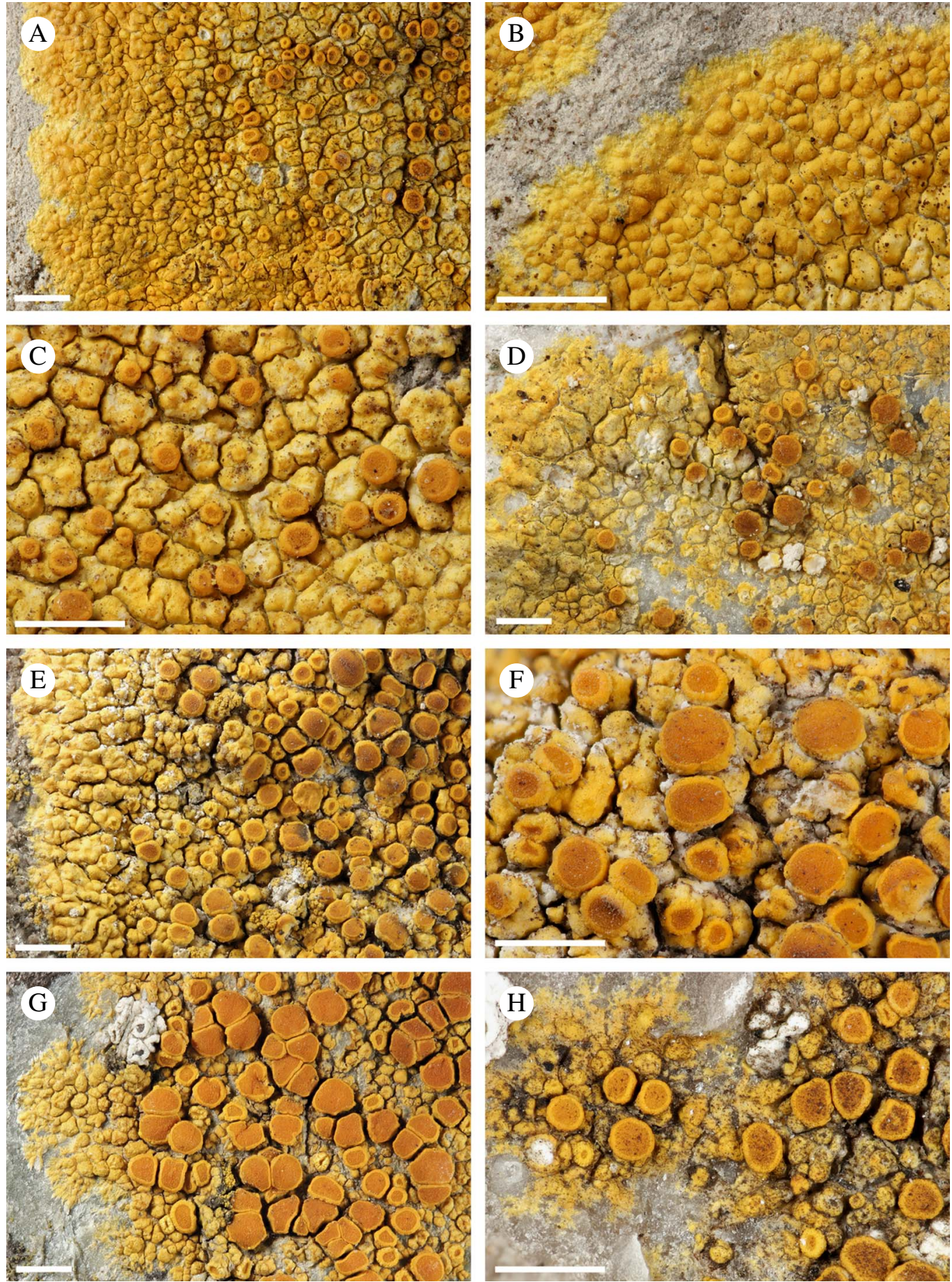

Fig. 2. A-F, Caloplaca sol. A-C, holotype; D, Orange 21068; E \& F, Orange 21916. G \& H, Caloplaca maritima; $\mathrm{G}$, Orange 20529; H, Orange 21047. Scales $=1 \mathrm{~mm}$. 

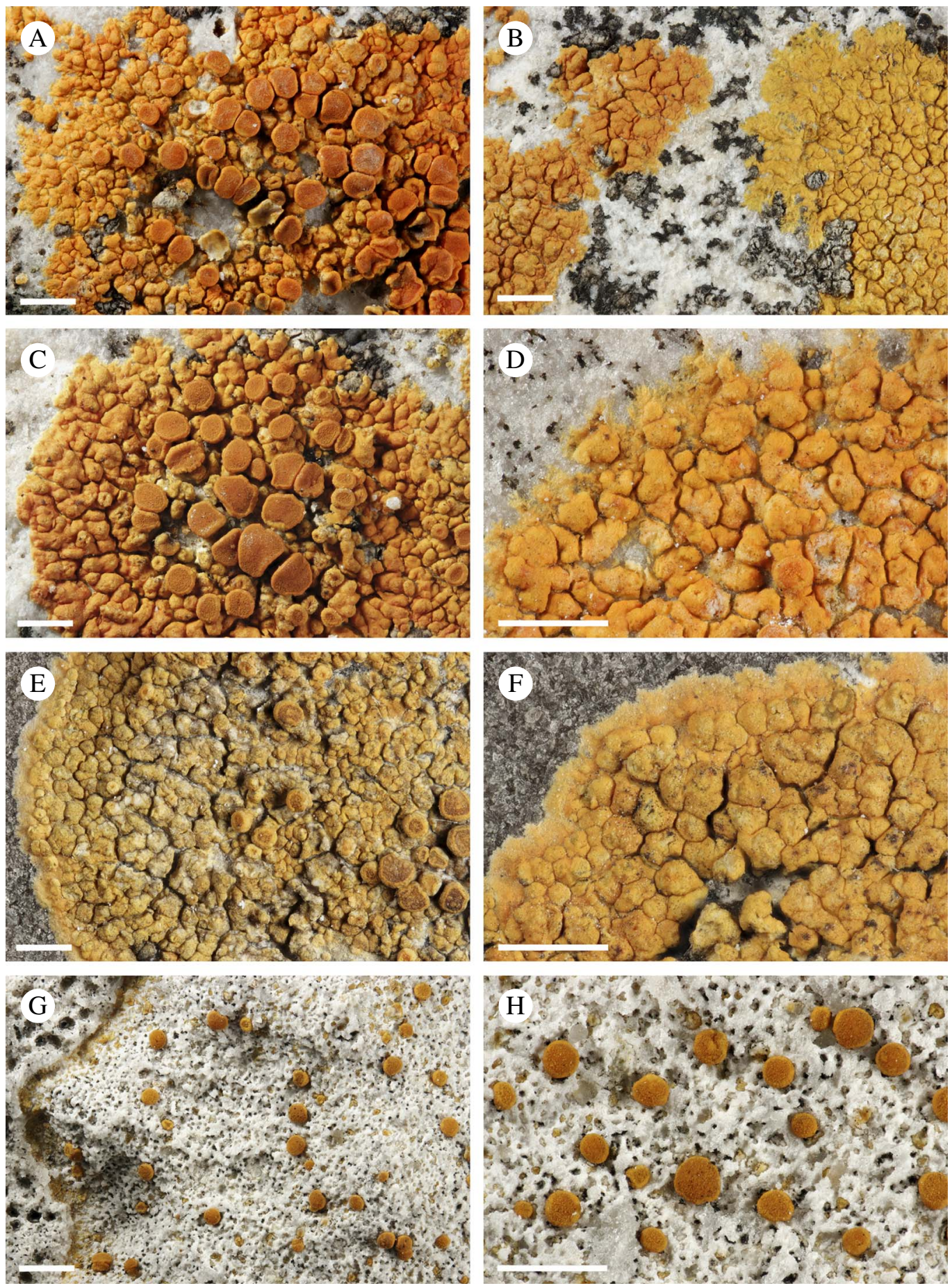

FIG. 3. A-F, Caloplaca marina; A-D, Orange 21071; B, C. marina (left) with C. sol (right). G \& H, Caloplaca dalmatica, Orange 21073. Scales $=1 \mathrm{~mm}$. 
1920, W. Watson (BM). V.C. 9, Dorset, Isle of Portland, below Grove Cliff, grid ref. 30/6991.7120, 50.539789 ${ }^{\circ} \mathrm{N}$, 02.425978 ${ }^{\circ} \mathrm{W}$, Orange 21062 (NMW-C.2015.005.39); Isle of Portland, below Grove Cliff, grid ref. 30/ $7047.7166,50 \cdot 543955^{\circ} \mathrm{N}, 02 \cdot 418112^{\circ} \mathrm{W}, 2012$, Orange 21068 (NMW-C.2015.005.42).

\section{Caloplaca dalmatica (A. Massal.) H. Olivier}

Mém. Soc. Sci. Nat. Cherbourg 37: 112 (1909).Callopisma dalmaticum A. Massal., Symmicta lichenum novorum vel minus cognitorum: 30 (1855); type: 'Legit in saxa in Dalmatia Cl. D. Lanza' (VER - not seen).

(Figs 3G \& H, 4A-D)

Prothallus endolithic, or epilithic, fimbriate, white, but very soon grading into the thin thallus margin; contiguous conspecific thalli sometimes separated by a groove (rarely discoloured by a superficial hyphomycete). Thallus almost completely endolithic, paler than surrounding rock, or locally pale yellow, or partly epilithic; often epilithic only at extreme margin, then pale orange-yellow (Figs 3G, 4A \& B), or epilithic thallus extensive, orange-yellow, typically discontinuous, entire or cracked, up to $220 \mu \mathrm{m}$ thick (Fig. 4C \& D). Apothecia soon sessile, to $0.4-0.6 \mathrm{~mm}$ diam., often evenly distributed but sometimes crowded; proper margin yellow-orange; thalline margin not developed, algal cells confined to lower side of apothecium; disc bright yellow when young, orange-yellow to orange when mature, sometimes yellow-pruinose, especially when young, plane to gently convex. Hymenium 70-80 $\mu \mathrm{m}$ high. Paraphyses 1.2$1.6 \mu \mathrm{m}$ wide near base, apical cells swollen, up to $8 \mu \mathrm{m}$. Asci 8-spored. Ascospores (8.6-) $11 \cdot 2-11 \cdot 8-12 \cdot 2(-18.0) \times(4 \cdot 9-) 6 \cdot 1-6 \cdot 4-6 \cdot 7(-$ $7.8) \mu \mathrm{m}, \mathrm{SD} \bar{x}_{2}=1.42$ and 0.60 respectively; length/width ratio $(1 \cdot 3-) 1 \cdot 7-1 \cdot 9-2 \cdot 0(-2 \cdot 5) \mu \mathrm{m}$, SD $\bar{x}_{2}=0 \cdot 23$; septum (4.1-)4.3-4.9-5.3(-7.4) $\mu \mathrm{m}, \mathrm{SD} \bar{x}_{2}=0.71$; septum/ascospore length $=(0.3-) 0.4-0.4-0.4(-0.5), \mathrm{SD} \bar{x}_{2}=0.05$. $\mathrm{N}=54, n=4$.

Pycnidia sometimes present near junctions of conspecific thalli. Conidia 3.3-3.7 × 1.2 $\mu \mathrm{m}$.

Ecology and distribution. On limestone, calcareous sandstone, mortar and plaster, often on steep or slightly overhanging faces, occasionally on gently inclined surfaces, in sun or light shade. Caloplaca dalmatica has been collected in the splash zone of the seashore, growing with $C$. marina, $C$. thallincola and Hydropunctaria maura, but most records are from well above the splash zone and it also occurs inland. Specimens for which ITS sequences are available were collected in North and South Wales, southern England (Dorset) and northern France.

Typification and nomenclature. The name Caloplaca dalmatica is used here in the sense of recent British lichen treatments (e.g. Fletcher \& Laundon 2009), and the application of this name is not investigated here. The type of Callopisma dalmaticum is in VER but is not available for loan (Francesco Di Carlo, in litt., 2015). The type locality is cited in Massalongo (1854: 19).

Notes. This species often has an almost completely endolithic thallus when growing on limestone but very often there is at least a narrow zone of thinly epilithic, yellow thallus at the growing margin. The endolithic thallus is typically paler than the surrounding rock and often appears whitish but frequently it is locally pale yellow. Occasionally, the thallus is thinly epilithic and sometimes cracked. Caloplaca sol differs in the well-developed, regularly cracked epilithic thallus. Caloplaca itiana differs in the less-developed thallus and the larger ascospores; on calcareous sandstone at the type locality of $C$. itiana, $C$. dalmatica has an epilithic, orange-yellow thallus (Fig. 4D) whereas $C$. itiana has at most a yellowish buff, very thin epilithic layer amongst the projecting sand grains of the substratum (Fig. 4E \& F). On limestone, based on the few specimens seen, $C$. itiana always has a completely endolithic thallus, with no trace of yellow thallus tissue (any yellow specks on the thallus are apothecial initials) (Fig. $4 \mathrm{H}$ ). Caloplaca navasiana is said to have an endolithic to thinly epilithic thallus but slightly smaller ascospores ((9.0-)10.0-11.7-13.0 $(-14.5) \mu \mathrm{m}$ long) and the apical cells of the paraphyses are slightly narrower $(3-6 \mu \mathrm{m}$ wide) (Navarro-Rosinés \& Roux 1995). 

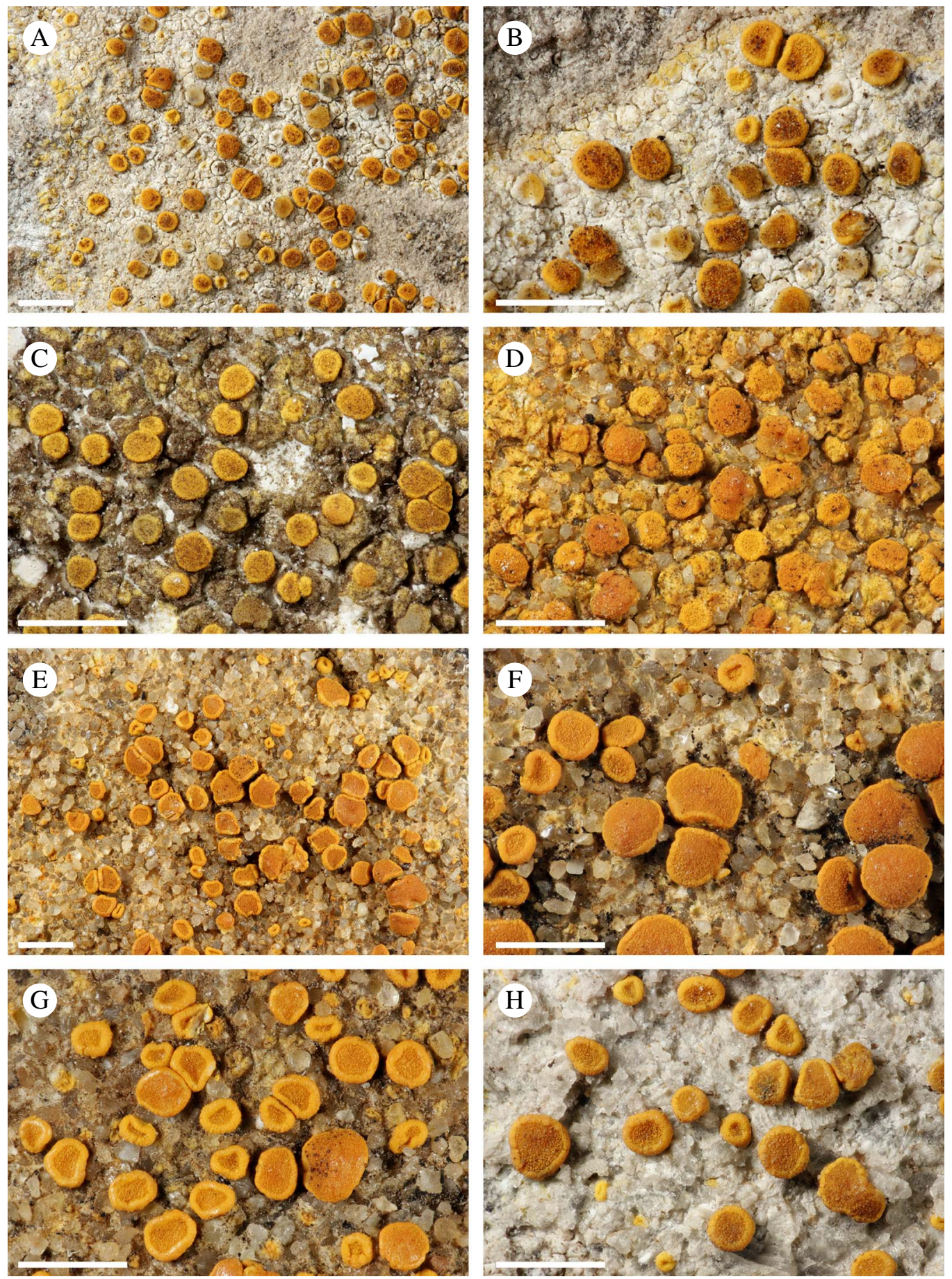

FIG. 4. A-D, Caloplaca dalmatica; A \& B, Orange 21053, growing on steep limestone; C, Orange 21076, on a limestone stone; D, Orange 23697, on calcareous sandstone. E-H, Caloplaca itiana; E \& F, Orange 23694, on calcareous sandstone (topotype); G, isotype (MARSSJ) on calcareous sandstone; H, Orange 21854, on limestone. Scales $=1 \mathrm{~mm}$. 
Selected specimens examined. Great Britain: Wales: V.C. 41, Glamorgan, Marcross, grid ref. 21/9210.6909, $51.410339^{\circ} \mathrm{N}, \quad 03.552916^{\circ} \mathrm{W}, 2012$, Orange 20900 (NMW-C.2017.005.1); Gower, Port Eynon, grid ref. $21 / 4657.8495,51.542522^{\circ} \mathrm{N}, 04.213922^{\circ} \mathrm{W}, 2014$, Orange 21766 (NMW-C.2015.005.54); Gower, Southgate, Foxhole, grid ref. 21/5546.8715, $51.564718^{\circ} \mathrm{N}$, $04.086517^{\circ} \mathrm{W}, 2014$, Orange 21803 (NMWC.2015.005.55); Gower, Southgate, Foxhole, grid ref. 21/ $5547.8721,51.565243^{\circ} \mathrm{N}, 04.086411^{\circ} \mathrm{W}, 2014$, Orange 21808 (NMW-C.2015.005.56). V.C. 52, Anglesey, Benllech, Trwyn Dwlban, grid ref. 23/5317.8201, $53.314746^{\circ} \mathrm{N}, \quad 04.206073^{\circ} \mathrm{W}, 2012$, Orange 21050 (NMW-C.2015.005.48); Benllech, grid ref. 23/ $5246.8237,53.317535^{\circ} \mathrm{N}, 04.216316^{\circ} \mathrm{W}, 2012$, Orange 21051 (NMW-C.2015.005.36); same locality and date, Orange 21053 (NMW-C.2015.005.38). England: V.C. 9, Dorset, Worth Matravers, Winspit, grid ref. 30/977.761, 1968, P. W. Fames (BM); north-west of Dorchester, Toller Porcorum, grid ref. $30 / 561.980,50 \cdot 779843^{\circ} \mathrm{N}, 02 \cdot 622385^{\circ}$ W, 2012, Orange 21057 (NMW-C.2015.005.49); Isle of Portland, below Grove Cliff, grid ref. 30/6991.7120, $50.539789^{\circ} \mathrm{N}, \quad 02.425978^{\circ} \mathrm{W}, 2012$, Orange 21060 (NMW-C.2015.005.50); Isle of Portland, below Grove Cliff, grid ref. $30 / 7017.7130,50 \cdot 540675^{\circ} \mathrm{N}, 02.42229^{\circ} \mathrm{W}$, 2012, Orange 21065 (NMW-C.2015.005.40); same locality and date, Orange 21067 (NMW-C.2015.005.41); same locality and date, Orange 21069 (NMWC.2015.005.51); same locality and date, Orange 21073 (NMW-C.2015.005.52); same locality and date, Orange 21076 (NMW-C.2015.005.41); Isle of Portland, north of Durdle Pier, grid ref. SY705.720, 1984, P. W. Fames 3037 \& V. F. Giavarini (BM).-France: Pas-de-Calais: Audinghen, Cap Gris-Nez, $50.87181^{\circ} \mathrm{N}$, $01.58601^{\circ} \mathrm{E}, 2017$, Orange 23695 (NMW-C.2017. 005.12); same locality and date, Orange 23697 (NMWC.2017.005.14); same locality and date, $50.87183^{\circ} \mathrm{N}$, $01.58523^{\circ} \mathrm{E}$, Orange 23699 (NMW-C.2017.005.16).

\section{Caloplaca itiana Cl. Roux, $M$. Boulanger \& Malle}

Bull. Ass. Fr. Lichénologie 34: 1-10 (2009); type: France, Pas-de-Calais, Audinghen, Boulonnais, Cap Griz-Nez, banc des Épaulards, 1 May 2008, C. Roux, M. Boulanger \& N. Malle (MARSSJ 23771-isotype!)

\section{(Fig. 4E-H)}

Prothallus not seen. Thallus endolithic, sometimes paler than surrounding rock, or very thinly epilithic as a discontinuous yellowish buff layer between projecting rock grains.

Apothecia sessile, $0.56-0.80 \mathrm{~mm}$ diam.; proper margin yellow-orange; disc plane, slightly darker than margin, eventually gently convex, sometimes yellow-pruinose when young; thalline margin not developed, algal cells confined to lower part of apothecium.
Paraphyses with the apical cell up to $8 \cdot 0(-10 \cdot 5)$ $\mu \mathrm{m}$ wide. Ascospores (10.7-)12.8-13.6-14.2 $(-16 \cdot 0) \times(5 \cdot 7-) 6 \cdot 8-7 \cdot 0-7 \cdot 3(-8 \cdot 6) \mu \mathrm{m}, \quad \mathrm{SD}$ $\bar{x}_{2}=1.34$ and 0.62 respectively; length/ width ratio (1.3-)1.8-2.0-2.0(-2.3), SD $\bar{x}_{2}=0 \cdot 24$; septum $(4 \cdot 1-) 4 \cdot 5-5 \cdot 0-5 \cdot 2(-5 \cdot 7)$, SD $\bar{x}_{2}=0.59 ;$ septum/ascospore length $=$ $(0 \cdot 3-) 0 \cdot 3-0 \cdot 4-0 \cdot 4(-0.5), \mathrm{SD} \bar{x}_{2}=0 \cdot 05 . \mathrm{N}=46$, $n=4$.

\section{Pycnidia not seen.}

Ecology and distribution. At the type locality the species is abundant on calcareous sandstone rocks on the upper seashore, especially on steeply sloping surfaces, very often mixed with Caloplaca dalmatica, but also on gently inclined surfaces where it was observed with C. thallincola and Hydropunctaria maura. Three specimens from Wales are from limestone on the coast, but distant from the spray zone, growing with terrestrial species including Caloplaca flavescens, Diplotomma alboatrum and Lecanora albescens.

Notes. This species was described by Roux et al. (2009) from calcareous sandstone on the seashore at Cap Gris-Nez in northern France, where it was found to form either monospecific stands or occurring mixed with Caloplaca thallincola, C. microthallina and Hydropunctaria maura. A visit to the type locality by the author revealed two frequent and morphologically quite similar species of Caloplaca growing together. In the field these differed mainly in the degree of development of the thallus, almost endolithic in one and thinly epilithic in the other. In addition, the apothecia of the endolithic taxon were slightly larger and darker than in the other. Microscopically they also differed in ascospore size and were very distinct in ITS sequence. Although the presence of two similar species occurring together at the type locality was not mentioned in the protologue, C. itiana clearly refers to the endolithic taxon, as an examined isotype is undoubtedly this species (Fig. 4G), accompanied by only a small thallus of the epilithic species, and the protologue refers to the endolithic or thinly epilithic thallus which is at most yellowish, unlike the distinctly yellow thallus 
of the epilithic taxon. The epilithic taxon is the species referred to as $C$. dalmatica in the present paper. The ITS tree suggests that Caloplaca itiana is closely related to $C$. calcitrapa and $C$. navasiana, but the tree does not provide a clear separation of these species. Caloplaca calcitrapa was described as having an epilithic thallus (Navarro-Rosinés et al. 2000) but according to Jan Vondrák (pers. comm.) a yellow thallus can be completely absent in $C$. calcitrapa. In the protologue, the ascospores were said to be of the "sand-clock" (hourglass) type, but Vondrák et al. (2009) found these in only a minority of specimens. There is a single British record of $C$. calcitrapa, on horizontal travertine of a balustrade; the thallus was said to be of yellow, bullate areoles (Powell \& Vondrák 2012). It seems likely that $C$. itiana is a synonym of $C$. calcitrapa but further study, perhaps using an additional gene region that is informative at the species level, is recommended before the synonymy is formally made. Caloplaca navasiana was described by Navarro-Rosinés \& Roux (1995); it was compared to C. itiana by Roux et al. (2009) and said to differ in the smaller ascospores and other minor characters. Of three available ITS sequences named as $C$. navasiana, one clearly represents a different taxon to the other two (Fig. 1), so at least one sequence is misidentified. For these reasons the status of this species deserves further study.

Three specimens from Wales with an endolithic thallus and relatively large ascospores are interpreted here as C. itiana, although only one exactly matches the ITS of two of the three sequenced topotypes. These specimens are less well developed and less conspicuous than material of $C$. itiana at the type locality; possibly calcareous sandstone is a more suitable substratum for this species than hard limestone. It is probable that this species has been overlooked or even ignored in Britain as the simple morphology and indistinct ascospores would have defeated attempts at certain identification using the literature available at the time. It is likely that specimens would have been included in a broad concept of $C$. holocarpa, a species now known to be only distantly related (Arup et al. 2013).
Caloplaca holocarpa s. str. occurs mainly on siliceous rock and the ascospores are smaller, (9.5-) 10.0-13.5(-15.5) $\mu \mathrm{m}$ according to Arup (2009). Caloplaca marmorata also has an endolithic thallus but differs in the markedly thin ascospore septum.

Additional specimens examined. Great Britain: Wales: V.C. 41, Glamorgan, Gower, Llanmadoc, Hills Tor, grid ref. $21 / 4284.9403,51.623084^{\circ} \mathrm{N}, 04.271724^{\circ} \mathrm{W}$, 2009, Orange 18786 (NMW-C.2011.014.37); Gower, Southgate, grid ref. $21 / 5587.8685,51.561828^{\circ} \mathrm{N}$, $04.080399^{\circ} \mathrm{W}, \quad 2014$, Orange 21854 (NMWC.2015.005.44). V.C. 45, Pembrokeshire, south of Pembroke, Stackpole National Nature Reserve, Merepool Valley, grid ref. 11/9740.9430, 51.610938 $\mathrm{N}$, $04.927415^{\circ} \mathrm{W}, \quad 2013$, Orange 21539 (NMWC.2013.001.96).-France: Pas-de-Calais: Audinghen, Cap Gris-Nez, $50 \cdot 87185^{\circ} \mathrm{N}, 01.58632^{\circ} \mathrm{E}, 2017$, Orange 23694 (NMW-C.2017.005.11); same locality and date, $50.87181^{\circ} \mathrm{N}, \quad 01.58601^{\circ} \mathrm{E}$, Orange 23696 (NMWC.2017.005.13); same locality and date, $50.87183^{\circ} \mathrm{N}$, $01.58523^{\circ} \mathrm{E}$, Orange 23700 (NMW-C.2017.005.17).

Claude Roux is thanked for his helpful response to questions concerning the type specimens of Caloplaca itiana. Jan Vondrák and Ulf Arup are thanked for reviewing the manuscript and for their helpful comments.

\section{REFERENCES}

Arup, U. (2006) A new taxonomy of the Caloplaca citrina group in the Nordic countries, except Iceland. Lichenologist 38: 1-20.

Arup, U. (2009) The Caloplaca holocarpa group in the Nordic countries, except Iceland. Lichenologist 41: 111-130.

Arup, U. \& Åkelius, E. (2009) A taxonomic revision of Caloplaca herbidella and C. furfuracea. Lichenologist 41: 465-480.

Arup, U., Søchting, U. \& Frödén, P. (2013) A new taxonomy of the family Teloschistaceae. Nordic fournal of Botany 31: 16-83.

Fletcher, A. \& Laundon, J. R. (2009) Caloplaca. In The Lichens of Great Britain and Ireland (C. W. Smith, A. Aptroot, B. J. Coppins, A. Fletcher, O. L. Gilbert, P. W. James \& P. A. Wolseley, eds): 245-273. London: British Lichen Society.

Frolov, I., Vondrák, J., Fernandez-Mendoza, F., Wilk, K., Khodosovsev, A. \& Halıc1, M. (2016) Three new, seemingly-cryptic species in the lichen genus Caloplaca (Teloschistaceae) distinguished in two-phase phenotype evaluation. Annales Botanici Fennici 53: 243-262.

Gaya, E., Högnabba, F., Holguin, Á., Molnar, K., Fernández-Brime, S., Stenroos, S., Arup, U., Søchting, U., van den Boom, P., Lücking, R., et al. (2012) Implementing a cumulative supermatrix approach for a comprehensive phylogenetic study of the Teloschistales (Pezizomycotina, Ascomycota). Molecular Phylogenetics and Evolution 63: 374-387. 
Guindon, S., Dufayard, J. F., Lefort, V., Anisimova, M., Hordijk, W. \& Gascuel, O. (2010) New algorithms and methods to estimate maximum-likelihood phylogenies: assessing the performance of PhyML 3.0. Systematic Biology 59: 307-321.

Lanfear, R., Calcott, B., Ho, S. Y. \& Guindon, S. (2012) PartitionFinder: combined selection of partitioning schemes and substitution models for phylogenetic analyses. Molecular Biology and Evolution 29: 1695-1701.

Lanfear, R., Frandsen, P. B., Wright, A. M., Senfeld, T. \& Calcott, B. (2017) PartitionFinder 2: new methods for selecting partitioned models of evolution for molecular and morphological phylogenetic analyses. Molecular Biology and Evolution 34: 772-773.

Massalongo, A. (1854) Geneacaena Lichenum. Veronae: Typis Ramanzinianis.

Miller, M. A., Pfeiffer, W. \& Schwartz, T. (2010) Creating the CIPRES Science Gateway for inference of large phylogenetic trees. In Proceedings of the Gateway Computing Environments Workshop (GCE), 14 November 2010, New Orleans, Louisiana, pp. 1-8.

Navarro-Rosinés, P. \& Roux, C. (1995) Caloplaca navasiana Nav.-Ros. et Roux $\mathrm{sp}$. nov., espèce nouvelle de lichen du littoral Méditerranéen. Cryptogamie, Bryologie-Lichénologie 16: 89-97.

Navarro-Rosinés, P., Gaya, E. \& Roux, C. (2000) Caloplaca calcitrapa sp. nov. (Teloschistaceae) un nuevo liquen saxícolo-calcícola mediterráneo. Bulletin de la Société Linnéenne de Provence 51: 145-152.

Powell, M. \& Vondrák, J. (2012) Italian balustrade at Cliveden hosts Italian lichens? British Lichen Society Bulletin 110: 11-14.

Roux, C., Boulanger, M. \& Malle, N. (2009) Caloplaca itiana sp. nov., espèce nouvelle de lichen du littoral de la Manche (France). Bulletin d'Informations de l'Association Française de Lichénologie 34: 1-10.

Šoun, J., Vondrák, J., Søchting, U., Hrouzer, P., Khodosovtsev, A. \& Arup, U. (2011) Taxonomy and phylogeny of the Caloplaca cerina group in Europe. Lichenologist 43: 113-135.

Stamatakis, A. (2014) RAxML version 8: a tool for phylogenetic analysis and post-analysis of large phylogenies. Bioinformatics 30: 1312-1313.

Stamatakis, A., Hoover, P. \& Rougemont, J. (2008) A fast bootstrapping algorithm for the RAxML Web servers. Systematic Biology 57: 758-771.

Tamura, K., Dudley, J., Nei, M. \& Kumar, S. (2007) MEGA4: Molecular Evolutionary Genetics Analysis (MEGA) software version 4.0. Molecular Biology and Evolution 24: 1596-1599.

Vondrák, J., Ř́ha, P., Arup, U. \& Søchting, U. (2009) The taxonomy of the Caloplaca citrina group (Teloschistaceae) in the Black Sea region; with contributions to the cryptic species concept in lichenology. Lichenologist 41: 571-604.

Vondrák, J., Øíha, P., Redchenko, O., Vondráková, O., Hrouzek, P. \& Khodosovtsev, A. (2011) The Caloplaca crenulatella species complex; its intricate taxonomy and description of a new species. Lichenologist 43: 467-481.

Vondrák, J., Khodosovtsev, A., Šoun, J. \& Vondráková, O. (2012) Two new European species from the heterogeneous Caloplaca holocarpa group (Teloschistaceae). Lichenologist 44: 73-89.

Vondrák, J., Halıcı, M. G., Güllü, M. \& Demiirel, R. (2016) Taxonomy of the genus Athallia and its diversity in Turkey. Turkish fournal of Botany 40: 319-328. 